\title{
Effect of Peripheral Communication Pace on Attention Allocation in a Dual-Task Situation
}

\author{
Sofiane Gueddana ${ }^{1,2}$ and Nicolas Roussel ${ }^{1,2}$ \\ ${ }^{1}$ LRI - Univ. Paris-Sud \& CNRS, Orsay, France \\ ${ }^{2}$ INRIA, Orsay, France \\ \{gueddana, roussel\} @lri.fr
}

\begin{abstract}
Peripheral displays allow continuous awareness of information while performing other activities. Monitoring such a display while performing a central task has a cognitive cost that depends on its perceptual salience and the distraction it causes, i.e. the amount of attention it attracts away from the user's primary action. This paper considers the particular case of peripheral displays for interpersonal communication. It reports on an experiment that studied the effect of peripheral communication pace on subjects' allocation of attention in a dual-task situation: a snapshot-based peripheral monitoring task where participants need to assess the presence of a remote person, and a central text-correcting task against the clock. Our results show that the addition of the peripheral task caused a drop in the success rate of the central task. As the pace of snapshots increased, success rate decreased on the peripheral task while on the central one, success rate remained the same but failures to reply in time occurred more frequently. These results suggest that the increase in pace of snapshots caused participants to change their strategy for the central task and allocate more attention to the peripheral one, not enough to maintain peripheral performance but also not to the point where it would affect central performance. Overall, our work suggests that peripheral communication pace subtly influences attention allocation in dual-task situations. We conclude by discussing how control over information pace could help users of communication systems to adjust their local distraction as well as the attention they draw from remote users.
\end{abstract}

Keywords: Computer-mediated communication, peripheral communication, attention allocation.

\section{Introduction}

Specific characteristics of interpersonal communication technologies have been studied for quite a while. Studies of video-mediated communication revealed few objective advantages of adding video to audio for focused problem solving tasks [1], for example, but they also showed the value of video for creating shared workspaces and assessing the availability of others. Media space studies particularly demonstrated this last role, emphasizing the importance of long-term and always-on connections and promoting the concept of peripheral awareness of each others' activities [2, 3]. Many of today's communication systems build on these notions of constant accessibility and 
peripheral awareness. A few additionally support the transformation of a peripheral communication into a primary activity. As an example, instant messaging applications not only provide constant information about people's presence and availability but also support the rapid exchange of text messages. Yet, managing users' transitions between background and foreground activities remains a key challenge of modern communication system design. In Weiser and Brown's terms, the challenge is to create calm technologies that engage both the center and the periphery of our attention and move back and forth between the two [4].

Monitoring a peripheral communication while performing another task has a cognitive cost that depends on the perceptual salience of the communication and the distraction it causes. Minimizing this cost is usually desirable and even necessary in situations like car driving where the ability to keep a communication in the background can be critical. But what appears as a cost might quickly turn into a benefit as one starts focusing on the peripheral communication and placing it at the center of attention. This paper considers the particular case of peripheral displays for interpersonal communication. We conducted a quantitative experiment to determine the effect of a snapshot-based peripheral monitoring task on subjects' attention in a dual-task situation. Our results suggest that peripheral communication pace subtly influences attention allocation in this situation. The paper is organized as follows. After introducing some related work, we describe the quantitative experiment that we conducted, summarize our results and conclude with a discussion.

\section{Related Work}

Attention is defined by psychologist William James as "the taking possession by the mind, in clear and vivid form, of one out of what seem several simultaneously possible objects or trains of thought" [5]. James further explains: "Focalization, concentration, of consciousness are of its essence. It implies withdrawal from some things in order to deal effectively with others, and is a condition which has a real opposite in the confused, dazed, scatterbrained state which in French is called distraction". As we said, a key challenge of modern communication system design is to create systems that do not monopolize user attention but can fade into the background and be promoted on demand to the foreground.

\subsection{Fading into the Background: Peripheral Awareness}

Communication systems designed to fade in the background avoid explicit interaction with users to prevent distraction. They instead resort to presets and automatic capture to determine the information to be communicated. Images, sounds and other automatically captured data can provide numerous awareness cues in this context, but at the cost of introducing privacy concerns. Filtering techniques have been proposed to help users mitigate these concerns by altering the data to be transmitted $[6,7,8]$ or by abstracting it to communicate higher-level information $[9,10,11]$.

Peripheral information displays also impose constraints on information rendering. As reported by Pousman and Stasko [12], numerous researchers have investigated ways to create systems that "aim at presenting information in a way that is not 
distracting but aesthetically pleasing and tangible to varying degrees". Information salience plays an important role in this context, defined as "the distinct subjective perceptual quality which makes some items in the world stand out from their neighbors and immediately grab our attention" [13]. Lights, haptics, scent and adapted everyday objects have been used to create low-salience ambient devices that blend into the user's environment $[14,15,16]$. Outside the specific domain of computer-mediated communication, studies have also investigated how to design peripheral displays so that they provide the most information while having the least impact on the user's performance on a primary task $[17,18,19]$. These studies particularly showed the ambivalent role of animations and the importance of physical characteristics of the display such as its size, position and orientation.

As illustrated by the previous examples, there is a substantial body of literature describing ambient communication devices or ambient information systems that might be used for communication. But creating systems that do not monopolize user attention addresses only the first part of the calm technology challenge. These systems should also be capable of promoting on demand interaction to the foreground. Unfortunately, the systems designed for background use can rarely be used for focused interaction, and conversely.

\subsection{From Background to Foreground}

A number of studies have investigated ways to help people maintain awareness of backgrounded tasks and facilitate their resumption [20]. Peripheral displays are known to help both interrupters to time communication and interruptees to handle interruptions $[21,20]$. But the transition of background tasks to the foreground requires that the displays incorporate mechanisms for drawing users' attention. A too low salience might lead them to miss important information or opportunities for more focused interactions, compromising coordination mechanisms. It has thus been suggested that systems should support different degrees of salience [9] and could increase the one of incoming messages that may deserve immediate attention [22]. People's attentional focus is known to be vulnerable to certain kinds of stimuli [23], and numerous techniques have been proposed that take advantage of this for getting users' attention [20].

The initiation of a communication is a gradual process that involves negotiation between parties [24]. This idea of gradual and negotiated engagement is also one of the most interesting aspects promoted by early media space studies $[25,26]$. As noted by Birnholtz et al., "paying attention to someone is itself a communicative act - an implicit request for interaction" and "interest in interaction on the part of the initiator is expressed by paying attention to his or her target in progressively more intrusive ways" [27]. Yet, relative little effort has been made to explore ways to actually support this gradual intrusion and the overall collaborative process of contact negotiation [28, 26], Community Bar [29, 30] and OpenMessenger [27, 31] being two notable recent exceptions.

Prior research has shown the value of peripheral displays as ways to maintain awareness of backgrounded tasks and facilitate their resumption. But monitoring a peripheral display while performing another task has a cognitive cost that system designers need to carefully take into account. The cost should be generally low, so 
that the display can fade into the background, but not too low to preserve its function. At times, a higher cost might be beneficial to get user's attention or in response to his or her increased interest as (s)he wants to promote the background task to the foreground. But how can we measure the resources used by a peripheral task?

\subsection{Measuring Attention}

The attentional cost of a particular task cannot be measured directly. Psychology studies of divided attention have thus mainly focused on dual-task situations in which participants share their limited attention between a main (or central) task and a peripheral one. When execution of at least one of the tasks deteriorates if they are performed simultaneously rather than separately, the tasks are said to interfere with each other: their combined attention requirements exceed resource capacity and execution deterioration in one can be used as a measure of the attentional cost variation of the other. Task execution deterioration is generally measured as a performance decrease, but can also be measured as an error rate or response time increase.

The idea that attention is sharable and limited was introduced by Kahneman, who also suggested that humans change their allocation policy over time [32]. According to his theory, although attention capacity is limited, it fluctuates according to arousal levels for internal causes (e.g. effort and motivation) and external ones (e.g. saliency of environment stimulation). Studies showed that motivational variables do not necessarily correlate with performance $[33,34]$. Other studies have illustrated the importance of three other factors on dual-task performance: task difficulty $[35,36]$, practice [37, 38] and task similarity [39]. Overall, these studies suggest that the similarity, novelty and complexity of two tasks greatly contribute to the fact that they compete for the same attentional resources.

Studies of peripheral displays have often used the dual-task paradigm to measure their attentional cost by comparing single and dual task execution. Our motivation for this work was a bit different. Having a significant experience in the design of videomediated communication systems, we were interested in attention allocation when peripherally monitoring regularly updated snapshots of a distant person. To our knowledge, the attentional cost of this task had never been formally evaluated. We were of course interested in measuring it, but we were more particularly interested in studying the effect of display richness on attention management. Dabbish and Kraut had already shown that monitoring an information-rich display imposes a substantial attentional cost and that an abstract display provides similar benefit with less distraction [40]. The information richness of regularly updated snapshots being somewhat difficult to control, we decided to focus on the pace of the update.

\section{Experiment}

We conducted a dual-task experiment with the following goals:

- (intermediate goal) determine the cost of adding a snapshot-based peripheral monitoring task to a central one;

- (main goal) determine the effect of varying the pace of snapshots on attention allocation. 
Measuring peripheral attention requires the peripheral and central tasks to be sufficiently novel, complex and similar to interfere with each other. The nature of the central task does not really matter as long as it interferes enough with the peripheral one. To test whether our two tasks indeed interfered and to formally evaluate the attentional cost of adding the snapshot-based peripheral monitoring task to the central one, we decided to compare single and dual task execution. Assuming that increasing the pace of snapshots would make the peripheral monitoring task more difficult, we were expecting the allocation of more attention to it and possibly the deterioration of the execution of one or both tasks. To precisely determine this effect, we decided to compare two different pace conditions and to measure execution deterioration in terms success, fail and timeout rates. Our hypotheses were the following:

- the addition of the snapshot-based peripheral monitoring task to a central one would have an effect on central task execution;

- varying the pace of snapshots would have an effect on the execution of both tasks.

\subsection{Tasks}

Participants had to simultaneously monitor a peripheral display while performing a central task. The central task and the peripheral one appeared on two separate screens. Inspired by the one used by Maglio and Campbell in a similar experiment [17], our central task was a text correcting exercise. This task involved mainly linguistic capabilities associated to decision making. To make the cognitive load adjustable, we introduced a time limit per sentence. By limiting the time available to read and decide, we turned the task into a "race against the clock" similar to the Tetris game used by Bartram et al. [41]. The top-right image of Fig. 1 shows the text correcting display. The sentence to correct is shown highlighted. At the top of the screen, a progress bar shows the time left to answer. At the bottom, two "correct" and "incorrect" buttons can be clicked with the mouse to provide the answer. Each text correction trial of the experiment, i.e. each sentence, had one among three outcomes: participants either clicked one of the buttons or didn't click any in time. The text used for the correcting task consisted in French articles with similar readability levels taken from a popular newspaper (Kandel and Moles measure [42] ranging from 48 to 52). Errors of two types were created by hand: syntax errors based on word inversions, and gender or number agreement errors. Zero, one or two errors were randomly introduced in each sentence of the articles presented to the participants.

For the peripheral task, we decided to simulate the peripheral awareness of distant people through an image-based communication system. Still pictures showing a desk and either a person or an empty chair were displayed for a random period of time on a separate screen, four seconds fade-in transitions being used between pictures (Fig. 1). We chose a monitoring rather than awareness task so that it would be sufficiently demanding and thus induce a dual-task performance trade-off. We also made this task more difficult in two ways: participants received instructions that emphasized the priority of the peripheral task over text correction, and changes in the remote person's presence as well as interruptions were very frequent. During the experiment, participants were randomly interrupted and both displays suspended at frequent times, i.e every 4 to 12 seconds. To measure monitoring performance in dual-task conditions, participants were then prompted to recall the presence state of the remote person by 


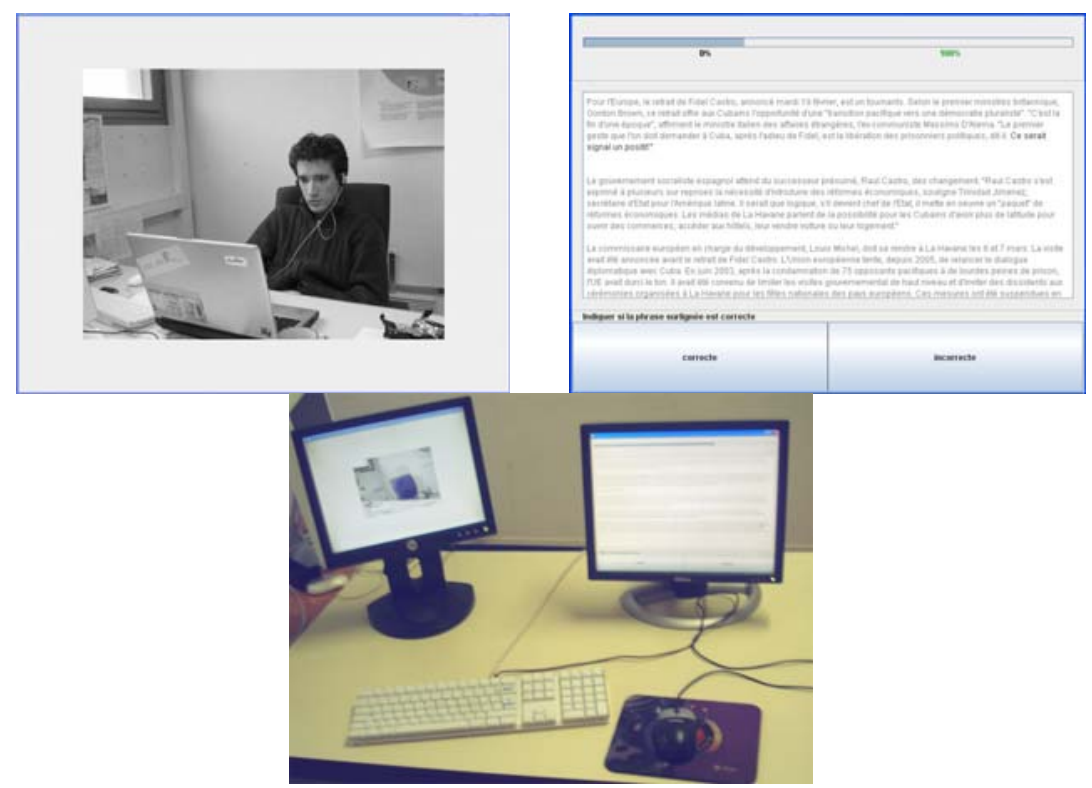

Fig. 1. Experiment tasks and apparatus

pressing the Return or Backspace key on a keyboard. To avoid introducing a bias when performing text correction alone, participants were also randomly interrupted in the single task condition, but simply had to press a key to resume it.

\subsection{Design}

The experiment was constructed as a one factor within subject design with a slow update pace condition, a fast update pace condition and a single task condition. Snapshots update pace ranged from 5 to 8 seconds in the fast condition, and from 17 to 21 seconds in the slow one. Executions of both tasks were compared across the update pace and single task conditions. Participants performed the single task condition as a control for the two dual-task conditions. The single task consisted in correcting a text document on a central screen with no peripheral task, while the dual-tasks included monitoring the presence of a remote person through snapshots displayed on a peripheral screen. Each participant performed both dual-task conditions and the single task condition.

The presentation order of conditions was balanced across two groups of participants (Fig. 2). The presentation order of articles in the text correction task was also balanced across participants. Dual-task trials were grouped in two blocks of 75 trials each, one for the fast condition and the other for the slow one. The single task condition was divided into three short blocks of 25 trials: before, between and after each dual-task condition block. 


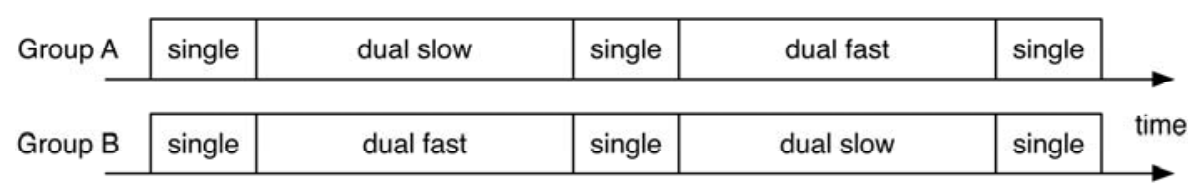

Fig. 2. Presentation order of dual-task condition blocks were counterbalanced in two groups, A and B. Single task blocks were interleaved with slow and fast blocks.

For presence monitoring, success rate (i.e. the percentage of correct recalls) was measured as a dependent variable. For text correction, each trial outcome was classified into one of three categories: correct, incorrect or failure to reply in time. Three dependent measures were then collected for each participant:

- success rate: the percentage of correct answers among all text trials;

- fail rate: the percentage of incorrect answers among all text trials;

- timeout rate: the percentage of failure to reply in time among all text trials.

\subsection{Procedure and Participants}

The experiment procedure included several phases: training, calibration, test and a post-test questionnaire. The task was orally briefly described, and detailed written instructions were displayed. These instructions emphasized the priority of monitoring the distant colleague over the text correction task. Participants then performed the three conditions during a training phase of 10 minutes. During this training, a visual feedback was displayed helping the participants learn the different error types. After the training phase, as text correction speed differed among participants, a speed calibration test of 15 minutes was performed. During this test, we varied the time per sentence to determine the appropriate speed for each participant to correctly perform the text correction. After calibration, the time limit was introduced and the test started. The participants performed the single task and dual-task blocks. After completing all blocks, participants answered a post-test questionnaire in two parts: participants had first to estimate their linguistic and visual abilities, and then to describe their subjective perception of the differences between the conditions and the strategies they employed in multitasking.

Twelve adult native French speakers were recruited for the experiment, ten males and two females aged from 22 to 30 (26 on average). All had normal or correct-to normal vision acuity and perimeter (more than $175^{\circ}$ ), six of them wearing lenses or glasses. The experiment apparatus consisted of two screens, a computer, a mouse and a keyboard. A distance of $20 \mathrm{~cm}$ separated the screens, two 1280x1024 LCD monitors (17" for the central and 15" for the peripheral), as seen at the bottom of Fig. 1. The software was implemented in Java 1.4 on a $3,4 \mathrm{GHz}$ Pentium 4 computer running Microsoft Windows XP Pro. Snapshots were 512 pixels wide by 384 pixels tall, scaled down from pictures taken at a resolution of 2560x1920 pixels. The text to correct was displayed using the Lucida Grande font in 13 points.

\subsection{Results}

Data was analyzed to determine the effect of adding the peripheral task to the central one and the effect of varying the pace of snapshots on both tasks. We compared 
success rate differences in peripheral monitoring under the two dual-task conditions (slow and fast) using a repeated measures analysis of variance (ANOVA). The test shows a significant difference of the success rate in the monitoring task between the slow and fast conditions $(\mathrm{F}=22.02, \mathrm{p}<.0001 *)$. In the slow condition, this rate was at an average of $96,85 \%$, while in the fast one, it fell to $92,78 \%$ (Fig. 3). We also looked at differences in the central task dependent variables under the three conditions (single, slow and fast) using ANOVA. We found a significant effect of condition on the success rate $(\mathrm{F}=9.53, \mathrm{p}<.0001 *)$ and timeout rate $(\mathrm{F}=12.82, \mathrm{p}<.0001 *)$, but not on the fail rate (Fig. 3). Concerning the success rate, the test shows a significant difference between single and dual-task conditions $\left(\mathrm{F}=9.35, \mathrm{p}=.0023^{*}\right)$, but no difference was found between the two pace conditions. For the timeout rate, we found no difference between the slow and single conditions, but the test shows a significant difference between the slow and fast conditions $(\mathrm{F}=12.8, \mathrm{p}<.0001 *)$.

Participants reported in the post-test questionnaires that they continued to learn multitasking and each task after the training period. We thus conducted a post-hoc analysis to evaluate learning, tiring, order and group effects on text correction. We found no significant difference in the success rate of the text correction task between the three single condition blocks. We also compared the first dual-task block with the second one for differences in text correction and monitoring success rate. The test shows significant differences between the two dual-task block for success rate of both the correction $\left(\mathrm{F}=5.53, \mathrm{p}=.0188^{*}\right)$ and the monitoring $\left(\mathrm{F}=5.19, \mathrm{p}=.0227^{*}\right)$ tasks. From the first to the second dual-task block, correction success rate rose from $69 \%$ to $74 \%$, while monitoring success rose from $94.0 \%$ to $95.9 \%$. Additionally, to evaluate order and group effect, we compared groups $\mathrm{A}$ and $\mathrm{B}$ in dual-task conditions for differences in success rate on both tasks. But we found no significant difference in the success rates, neither for correction nor for monitoring. We also compared groups A and B in

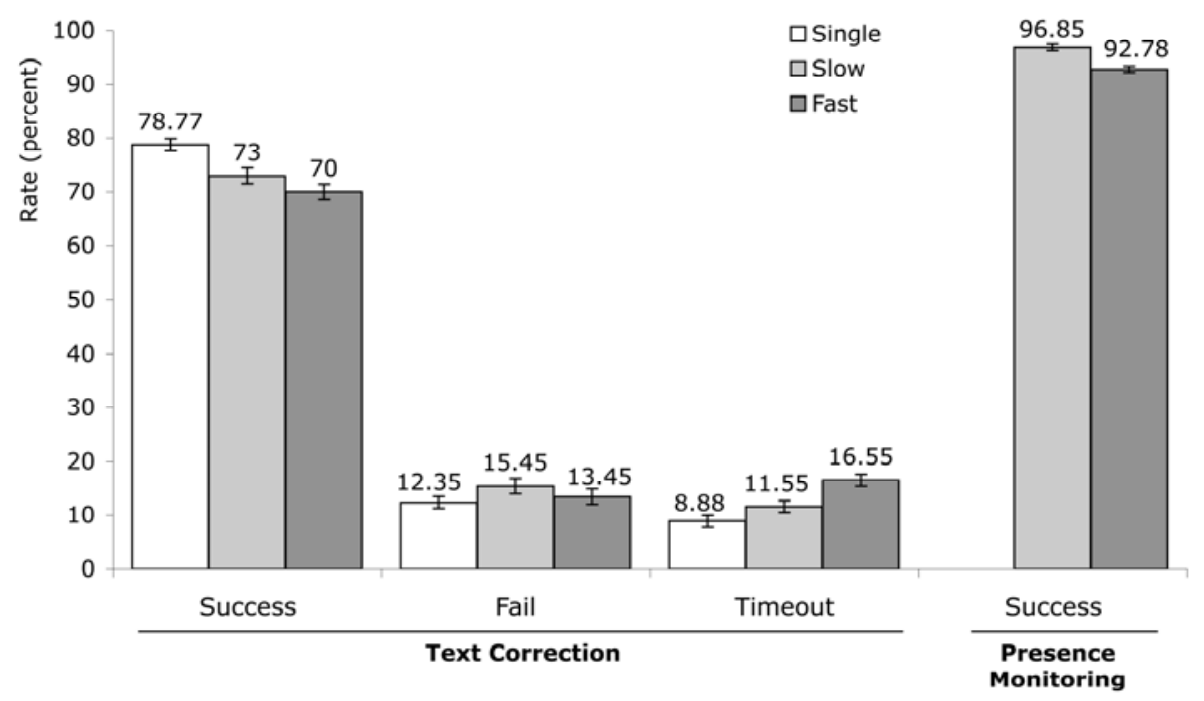

Fig. 3. Text correction and monitoring performance across the three conditions. Error bars represent standard error. 
Table 1. Two by two comparison across all conditions using ANOVA test of the dependant variables: monitoring success rate, success and text correction timeout rate

\begin{tabular}{llll}
\hline Conditions & Monitoring success & Correction success & Correction timeout rate \\
\hline slow vs. fast & $\mathrm{F}=22.02, \mathrm{p}<.0001^{*}$ & no difference & $\mathrm{F}=12.8, \mathrm{p}<.0001^{*}$ \\
single vs. fast & - & $\mathrm{F}=18.71, \mathrm{p}<.0001^{*}$ & $\mathrm{~F}=24.37, \mathrm{p}<.0001^{*}$ \\
single vs. slow & - & $\mathrm{F}=8.46, \mathrm{p}=.0037^{*}$ & no difference \\
\hline dual-task first vs. & & & \\
second blocks & $\mathrm{F}=5.19, \mathrm{p}=.0227^{*}$ & $\mathrm{~F}=5.53, \mathrm{p}=.0188^{*}$ & $\mathrm{~F}=4.42, \mathrm{p}=.0355^{*}$ \\
single task blocks & - & $\mathrm{F}=8.46, \mathrm{p}=.0037^{*}$ & no difference \\
\hline
\end{tabular}

the single task condition and did not find any significant difference in the success rate of the correction task.

Table 1 summarizes the results of our tests comparing success rate in the monitoring task, and success and timeout rates in the text correction task. The upper part shows the results of the comparison of the slow, fast and single conditions and illustrates the effect of pace variation and peripheral task addition. The lower part shows the results of the comparison of dual-task and single task blocks and illustrates the learning effect.

\subsection{Results Analysis}

Our results show that the addition of the peripheral monitoring task caused a drop in the success rate of the central task. Subjective data gathered from the post-test questionnaires showed that participants perceived this negative effect. While we found no significant difference in the success rate of the central task between the slow and fast conditions, the timeout rate increased with the pace of snapshots. Four participants reported through the questionnaires that they adapted their text correction strategy according to their free time and tried to schedule their glances towards the peripheral display so as to minimize performance loss on the central task. Overall, these results suggest that increasing the pace of snapshots caused participants to subtly change their text correction strategy. The increase of the timeout rate with the pace of snapshots suggests that participants allocated more attention to the peripheral monitoring task, although not to the point where it would have significantly affected central performance.

Increasing the pace of snapshots led to a significant drop in the success rate of the peripheral task. Data from the questionnaires further indicates that this performance drop was not perceived by seven out of the twelve participants. This suggests that participants did not allocate enough attention to the peripheral task in the fast condition. Combined with the fact that no significant difference was found in the success rate of the central task between the slow and fast conditions, this suggests that participants did not observe the peripheral task priority emphasized in the instructions they received; although the attention required by the peripheral task increased with the pace of snapshots, participants contained this task at the periphery.

Results of the tests on group and order show no effect on both tasks, suggesting that groups A and B are not intrinsically different. Comparison of blocks in the dualtask conditions shows a significant positive change in the success rate of the central 
task over time, which suggests a learning effect. Participants indeed reported through the questionnaires that they continued to learn how to perform both tasks and to multitask them after the training period. However, comparison of the blocks in the single condition doesn't show any change in the success rate of the central task, which suggests that the learning effect observed on the central task was probably related to better multitasking and not better task performance. Comparison of blocks in the dualtask conditions also shows a significant positive change in the success rate of the peripheral task over time. But in this case, it is unclear whether participants learned to better perform the monitoring task or again benefited from improved multitasking skills.

\section{Discussion}

"When our periphery is functioning well we are tuned into what is happening around us, and so also to what is going to happen, and what has just happened. (...) The periphery connects us effortlessly to a myriad of familiar details." M. Weiser and J. S. Brown, The coming age of calm technology, 1996

"There ain't no such thing as a free lunch"

P. Dos Utt, TANSTAAFL: a plan for a new economic world order, 1949

As far as snapshot-based peripheral displays are concerned, our experiment contradicts Weiser and Brown's dream of an effortless connection to the periphery and rather support Dos Utt's idea: the addition of the peripheral monitoring task caused a drop in the success rate of the central task. This corroborates in the particular case of social awareness the findings of Maglio and Campbell who also saw a negative effect on the performance of a central task when adding a peripheral one [17], and contradicts the results of Plaue and Stasko who saw no effect on their central task execution [43]. But as explained by McFarlane and Latorella, understanding human performance with interruptions is a complex problem [20]. As an example, Mark et al. recently showed that interrupted work can sometimes be performed faster, at the cost of a higher workload, more stress, higher frustration, more time pressure and effort [44]. It was not the case in our experiment. More work is certainly needed to better understand the induced costs and benefits of the various kinds of peripheral displays.

Training has long been known as a way to reduce the negative effects of interruptions [45]. As we explained, participants of our experiment reported that they continued to learn how to perform both tasks and to multitask them after the training period, the latter being confirmed by our quantitative results. This corroborates the results of Wickens and Damos that indicated that timesharing skills improve with practice, reducing the interference between the peripheral and the central task [37]. The cognitive resources involved in the peripheral task can also modulate its effects. As an example, the peripheral task used by Maglio and Campbell [17] was a ticker memorization task, and no difference was found among the displays (tickers) in memorability. In our case, the success rate of the peripheral task was indeed affected by the pace of the snapshots. A third factor that can also modulate the effects of the peripheral task is the interaction modalities it uses, which can more or less conflict with those of the central task $[46,20]$. In our case, both tasks were intrinsically visual. 
We believe the effects we observed of peripheral update pace on attention allocation are not limited to our experimental conditions. These effects can probably be generalized to other conditions, like awareness tasks rather than monitoring tasks. According to McCrickard et al. [18], an awareness question consists in asking participants to recall some information that had been displayed, as in our task. Plaue and Stasko explain that motivation makes the difference between awareness and monitoring tasks [43]. In that sense, our peripheral task was a (motivated) monitoring task. But we argue that in both motivated and non-motivated situations, the effort required to maintain peripheral attention is influenced by the update rate of the display. As we said, according to Kahneman [32], motivation is an important factor that influences attention capacity and allocation policy. In the case of (non-motivated) awareness, the peripheral task might simply be ignored by participants in which case no noticeable interference on the central task would probably be found.

Results from our experiment show that increasing the pace of snapshots increased the attentional cost of the peripheral task. Reducing the pace should reduce distraction and thus help keep the task in the background. However, it is unclear whether further increasing the pace would end up promoting the peripheral task to the foreground, or whether users would manage to contain it at the periphery, at the price of lowering performance. Future studies should investigate this. In any case, from a broader perspective, precise control over the update rate of a peripheral display might be a good way of both ensuring that the associated task remains in the background and, at times, initiate a gradual attention shift.

\section{Conclusion and Future Work}

Monitoring a peripheral display while performing another task has a cognitive cost. This paper reported on a quantitative experiment that we conducted to determine the effect of a snapshot-based peripheral monitoring task on subjects' attention in a dualtask situation. Our results show that the addition of the peripheral task caused a drop in the success rate of the central task. They also suggest that the increase in pace of snapshots caused participants to change their strategy for the central task and allocate more attention to the peripheral one, not enough to maintain peripheral performance but also not to the point where it would affect central performance. Overall, our work suggests that peripheral communication pace subtly influences attention allocation in dual-task situations.

While Romero et al. suggest that communication systems designers should strive for a better balance between distraction, awareness, and screen resources [30], we believe that users should be given the opportunity to negotiate this balance together. Deliberately raising the cognitive cost of a communication can be interpreted as an increased interest in it. By varying the pace of a communication, a user might hope gaining remote people's attention more easily and inciting them to engage a little further. As pointed out by Tang [28], current communication systems leave very little room for this kind of negotiation. Our work suggests that systems should allow the initiator to decide how important and salient a communication is, and not only the recipient. Pousman and Stasko also suggested that designers might start building systems supporting a range of notification levels and not just one [12]. We plan to create new communication systems prototypes to further explore these design spaces. 


\section{Acknowledgements}

This work has been supported by France Télécom R\&D as part of the DISCODOM project (2005-2008). We wish to thank our experiment participants and Wendy Mackay, Olivier Chapuis and the anonymous reviewers for their feedback.

\section{References}

1. Whittaker, S.: Rethinking video as a technology for interpersonal communications: theory and design implications. International Journal of Human-Computer Studies 42(5), 501-529 (1995)

2. Dourish, P., Adler, A., Bellotti, V., Henderson, A.: Your place or mine? Learning from long-term use of audio-video communication. Computer Supported Cooperative Work 5(1), 33-62 (1996)

3. Dourish, P., Bly, S.: Portholes: supporting awareness in a distributed work group. In: Proceedings of CHI 1992, pp. 541-547. ACM Press, New York (1992)

4. Weiser, M., Brown, J.S.: Designing calm technology. PowerGrid Journal 1(01) (1996)

5. James, W.: The principles of Psychology. Henry Holt (1890)

6. Hudson, S.E., Smith, I.: Techniques for Addressing Fundamental Privacy and Disruption Tradeoffs in Awareness Support Systems. In: Proceedings of CSCW 1996, pp. 248-257. ACM Press, New York (1996)

7. Zhao, Q., Stasko, J.T.: Evaluating Image Filtering Based Techniques in Media Space Applications. In: Proceedings of CSCW 1998, pp. 11-18. ACM Press, New York (1998)

8. Boyle, M., Edwards, C., Greenberg, S.: The effects of filtered video on awareness and privacy. In: Proceedings of CSCW 2000, pp. 1-10. ACM Press, New York (2000)

9. Greenberg, S., Kuzuoka, H.: Using digital but physical surrogates to mediate awareness, communication and privacy in media spaces. Personal and Ubiquitous Computing 3(4), 182-198 (1999)

10. Fogarty, J., Hudson, S.E., Atkeson, C.G., Avrahami, D., Forlizzi, J., Kiesler, S., Lee, J.C., Yang, J., Yang, J.: Predicting human interruptibility with sensors. Transactions on Computer-Human Interaction 12(1), 119-146 (2005)

11. Avrahami, D., Hudson, S.E.: Responsiveness in instant messaging: predictive models supporting inter-personal communication. In: Proceedings of CHI 2006, pp. 731-740. ACM Press, New York (2006)

12. Pousman, Z., Stasko, J.T.: A taxonomy of ambient information systems: four patterns of design. In: Proceedings of AVI 2006, pp. 67-74. ACM Press, New York (2006)

13. Itti, L.: Visual salience. Scholarpedia 2(9), 3327 (2007)

14. Strong, R., Gaver, B.: Feather, scent and shaker: supporting simple intimacy. In: Proceedings of CSCW 1996, pp. 29-30. ACM Press, New York (1996)

15. Brave, S., Ishii, H., Dahley, A.: Tangible interfaces for remote collaboration and communication. In: Proceedings of CSCW 1998, pp. 169-178. ACM Press, New York (1998)

16. Dey, A.K., de Guzman, E.: From awareness to connectedness: the design and deployment of presence displays. In: Proceedings of CHI 2006, pp. 899-908. ACM Press, New York (2006)

17. Maglio, P.P., Campbell, C.S.: Tradeoffs in displaying peripheral information. In: Proceedings of CHI 2000, pp. 241-248. ACM Press, New York (2000) 
18. McCrickard, D.S., Catrambone, R., Stasko, J.T.: Evaluating animation in the periphery as a mechanism for maintaining awareness. In: Proceedings of Interact 2001, pp. 148-156. IOS Press, Amsterdam (2001)

19. Campbell, C.S., Maglio, P.P.: Segmentation of display space interferes with multitasking. In: Proceedings of Interact 2003, pp. 575-582. IOS Press, Amsterdam (2003)

20. McFarlane, D.C., Latorella, K.A.: The scope and importance of human interruption in human-computer interaction design. Human-Computer Interaction 17(1), 1-61 (2002)

21. Dabbish, L., Kraut, R.: Controlling interruptions: awareness displays and social motivation for coordination. In: Proceedings of CSCW 2004, pp. 182-191. ACM Press, New York (2004)

22. Avrahami, D., Hudson, S.E.: QnA: augmenting an instant messaging client to balance user responsiveness and performance. In: Proceedings of CSCW 2004, pp. 515-518. ACM Press, New York (2004)

23. Muller, H.J., Rabbitt, P.M.A.: Reflexive and voluntary orienting of visual attention: Time course of activation and resistance to interruption. Journal of Experimental Psychology: Human Perception and Performance 15(2), 315-330 (1989)

24. Clark, H.H.: Using Language. Cambridge University Press, Cambridge (1996)

25. Gaver, W., Moran, T., MacLean, A., Lövstrand, L., Dourish, P., Carter, K., Buxton, W.: Realizing a Video Environment: EuroPARC's RAVE System. In: Proceedings of CHI 1992, pp. 27-35. ACM Press, New York (1992)

26. Roussel, N., Gueddana, S.: Beyond "Beyond being there": towards multiscale communication systems. In: Proceedings of Multimedia 2007, pp. 238-246. ACM Press, New York (2007)

27. Birnholtz, J.P., Gutwin, C., Hawkey, K.: Privacy in the open: how attention mediates awareness and privacy in open-plan offices. In: Proceedings of Group 2007, pp. 51-60. ACM Press, New York (2007)

28. Tang, J.C.: Approaching and leave-taking: negotiating contact in computer-mediated communication. Transactions on Computer-Human Interaction 14(1), a. 5 (2007)

29. McEwan, G., Greenberg, S.: Supporting social worlds with the community bar. In: Proceedings of Group 2005, pp. 21-30. ACM Press, New York (2005)

30. Romero, N., McEwan, G., Greenberg, S.: A field study of Community Bar (mis)-matches between theory and practice. In: Proceedings of Group 2007, pp. 89-98. ACM Press, New York (2007)

31. Birnholtz, J.P., Gutwin, C., Ramos, G., Watson, M.: OpenMessenger: gradual initiation of interaction for distributed workgroups. In: Proceedings of CHI 2008, pp. 1661-1664. ACM Press, New York (2008)

32. Kahneman, D.: Attention and effort. Prentice-Hall, Englewood Cliffs (1973)

33. Wickens, C.D.: Processing resources in attention. In: Varieties of Attention, pp. 63-102. Academic Press, London (1984)

34. Yerkes, R., Dodson, J.D.: The relation of strength of stimulus to rapidity of habit formation. Journal of Comparative Neurological Psychology 18, 469-482 (1908)

35. Sullivan, L.: Selective attention and secondary message analysis: A reconsideration of broadbent's filter model of selective attention. Quarterly Journal of Experimental Psychology 28(2), 167-178 (1976)

36. Duncan, J.: Divided attention: The whole is more than the sum of its parts. Journal of Experimental Psychology 5(2), 216-228 (1979)

37. Damos, D.L., Wickens, C.D.: The identification and transfer of timesharing skills. Acta Psychologica 46(1), 15-39 (1980) 
38. Spelke, E., Hirst, W., Neisser, U.: Skills of divided attention. Cognition 4(3), 215-230 (1976)

39. Treisman, A., Davies, A.: Divided attention to ear and eye. In: Kornblum, S. (ed.) Attention and performance IV, pp. 101-117. Academic Press, London (1973)

40. Dabbish, L., Kraut, R.: Coordinating communication: awareness displays and interruption. In: Proceedings of CHI 2003, pp. 786-787. ACM Press, New York (2003)

41. Bartram, L., Ware, C., Calvert, T.: Moticons: detection, distraction and task. International Journal of Human-Computer Studies 58(5), 515-545 (2003)

42. Moles, A., Kandel, L.: Application de l'indice de Flesch à la langue française. Cahiers d'Etudes de Radio-Télévision (19), pp. 252-274 (1958)

43. Plaue, C., Stasko, J.T.: Animation in a peripheral display: distraction, appeal, and information conveyance in varying display configurations. In: Proceedings of GI 2007, pp. 135142. ACM, New York (2007)

44. Mark, G., Gudith, D., Klocke, U.: The cost of interrupted work: more speed and stress. In: Proceedings of CHI 2008, pp. 107-110. ACM Press, New York (2008)

45. Hess, S.M., Detweiler, M.C.: Training to reduce the disruptive effects of interruptions. In: Proceedings of the Human Factors and Ergonomics Society 38th Annual Meeting, pp. 1173-1177 (1994)

46. Storch, N.A.: Does the user interface make interruptions disruptive?: a study of interface style and form of interruption. In: CHI 1992 extended abstracts, p. 14. ACM Press, New York (1992) 